\title{
Motion Platform Designed for Container Crane Simulator
}

\author{
${ }^{1,2}$ Houjun Lu, ${ }^{1}$ Huiqiang Zhen, ${ }^{2}$ Youfang Huang and ${ }^{2}$ Guihui Guo \\ ${ }^{1}$ School of Mechanical Engineering, Tongji University, Shanghai, 200092, China \\ smulhj@126.com \\ ${ }^{2}$ Container Supply Chain Engineering Research Center, Shanghai Maritime \\ University, Shanghai, 201306, China
}

\begin{abstract}
Container crane simulator is an important tool for driver training to increase the operation safety. Motion platform is always used for providing the motion perception for the crane simulator, and is also the critical approach to improve the simulation fidelity for the high-end simulator. Based on the designed parameters and the operation mode of the container crane, a novel design method of the container crane simulator motion platform is proposed in this work, which includes the work space design of mechanical component and the technical specifications analysis of the platform According to the principle of human-being's motion perception, we design the control wash-out algorithm which is the core part to determine the platform how to work.. We use the simulation tool to optimize the parameters of the filters and evaluate the performance of the motion platform whether can satisfy the requirement of crane simulator. Viewed from the running in the field, the motion platform has been developed completely and used for one type of the crane simulator, which validates the method proposed by this work that can be applied for the container crane simulator.
\end{abstract}

Keywords: container crane, simulator, motion platform, wash-out algorithm

\section{Introduction}

With the widely use of the quayside container crane in the loading and unloading operation in the port, the huge amount of the loading and uploading container, the increase of the machine efficiency, the improvement of the production safety and service life as well as the increase of economic benefit of the port all determine the importance of the training to the quayside container crane drivers. Because of the repeatability, safety, and the economical of the driving simulator, the problems of the drivers training can be easily effectively resolved. The motion platform as the hardware implementation of the simulator can't provide an enough route for the real quay crane moving in the simulating process, because of the limit of the motion platform structure. Then the motion platform needs to move back to the initial position [1] under the body feeling threshold value and provide an enough route for another simulation move. The back process is a spare motion. The Wash-out algorithm can solve the problem of the remove the body movement feeling of spare process.

The performance of the motion platform has a great influence on the simulation fidelity, and many scholars have done a lot of research work for the design and implementation of motion platform. Stewart [2] presented a 6-DOF parallel manipulator and design the control mode or strategies for the driving modes of the platform, and the parallel mechanism always can be used widely[10]. Robert J. Telban[3] designed a nonlinear control wash-out algorithm for the platform, which can generate serial insinuation and time-varying in the vertical mode through the real-time update algorithm parameters from the optimal control equation, cooperating with the optimum control law in the vestibular system model. Chung-Shu LIAO[4] mentioned a new algorithm for the 
6-DOF motion platform, who detected the boundary of the operating stroke and input the message into the filter, which made the movement hint could be provided in the finite space that guaranteed the robustness of system. Peter Grant et al.[5]analyzed the state of the actuator's hydraulic cylinder. He changed the algorithm design of legacy inertial frame, which can solve the cost function for the state of simulator. Wang Xiaoliang[6] studied the driving simulator of high speed train that the different fidelity was effected by the different wash-out location. Tang Yi[7] studied the vehicle simulator and presented that the implementation of the movement sense should fit the requirement of the programming. Wei Chunyu[8] et al. developed the Stewart platform and kinematics simulation by Vortex completed the visualize of wash-out process. Gao Jian[9] presented the identification method of the control behavior and movement match, and the design method is modified through reference fidelity estimate. The feasibility and veracity is verified in the taking off, cruise and landing condition.

Container crane simulator is used for simulating kinds of crane's handling process. Not only is it used for improving the driver's proficiency, but also it can simulate different parameters of apparatus' effect of dynamic capability. As the fidelity of visual and auditory improved in the simulator, it is an important way to increase simulator's performance by providing motion perception, especially for the advanced high-end simulator. Based on the particular container crane design parameters, we determine the technical specifications of platform and the process of wash-out algorithm by the mechanism analysis, kinematics analysis, and stability analysis and so on. The control wash-out algorithm of motion platform referred to the process design, filter's form and parameters adjustment, is described in detail in this work. As the simulation tool, we use Matlab Simulink to test how the different parameters' affect on the performance of motion platform.

\section{System Design of motion platform}

\subsection{Type of Crane Selected}

The type of container crane selected for the simulator is comprised of hoisting mechanism, pitching mechanism, crane traveling mechanism and trolley traveling mechanism. The motion platform is designed for providing the motion perception of the driver during the crane or trolley movement. The work space of the motion platform must be designed based on the crane's technical parameters, listed in table 1.

Table1. Parameters of the Crane Selected

\begin{tabular}{c|ll}
\hline Type & \multicolumn{1}{|c}{ Items } & Value \\
\hline \multirow{4}{*}{ Geometric Parameter } & Seaside Length & $70.5 \mathrm{~m}$ \\
& Landside Length & $23 \mathrm{~m}$ \\
& Gauge & $30.48 \mathrm{~m}$ \\
& Height & $40 \mathrm{~m}$ \\
\hline \multirow{2}{*}{ Dynamic Parameter } & The highest speed of crane & $45 \mathrm{~m} / \mathrm{min}$ \\
& The highest speed of trolley & $180 \mathrm{~m} / \mathrm{min}$ \\
\hline
\end{tabular}

\subsection{Technical Specifications}

Motion platform can provide the motion sense for the driver in the crane simulator. Based on the dynamics properties of the quay crane, the platform is designed to generate the motion signals of the instantaneous acceleration, sustained acceleration and so on in the simulator, which are as the same as possible with the real environment. The motion platform can provide the instantaneous acceleration of 
crane and trolley. In addition, the sustained acceleration can be compensated by pitching. We select those parameters as our technical specifications, such as full load weight, the pitch angle of the driver's cab on the motion platform, the instantaneous acceleration and instantaneous linear velocity of the crane and trolley, and the length of motion path, listed in table 2 .

Table 2. Technical Specifications of the Motion Platform

\begin{tabular}{l|l}
\hline Type & index \\
\hline Dynamic load & $2 \mathrm{~T}$ \\
Inclined angle & $\pm 6^{\circ}$ \\
Instantaneous acceleration of trolley & $\pm 0.5 \mathrm{~g}$ \\
Instantaneous acceleration of crane & $\pm 0.5 \mathrm{~g}$ \\
Instantaneous linear velocity of & $\pm 500 \mathrm{~mm} / \mathrm{s}$ \\
trolley & $\pm 500 \mathrm{~mm} / \mathrm{s}$ \\
Instantaneous linear velocity of crane & $\pm 80 \mathrm{~mm}$ \\
Length of motion path for trolley & $\pm 60 \mathrm{~mm}$ \\
Length of motion path for crane &
\end{tabular}

\subsection{Mechanical Structure}

The motion platform mentioned in this work is mainly composed of the upper frame and lower frame. An electric cylinder is installed between the upper frame and lower frame, which can control the movement system. The driver's cab is fixed on the upper frame. The lower frame is rectangle with welded steel tube, and fixed on the floor with bolts. There are 3 fixed bases for the electric cylinder in the lower frame. The upper frame is rectangle with welded steel tube, and locked together with the driver's cab. The driver's cab can move on the motion path. There are 2 lateral rails and 2 longitudinal rails fixed between the upper frame and lower frame, and the upper frame can move in two directions on the rails. The electric motion system is connected with cross-garnet butt. We finally choose the spherical bearing when compared with the others, and so the motion platform can pitch with the spherical bearing achieve the tilting compensate. Fig.1 presents the Mechanical structure of motion platform mechanism.

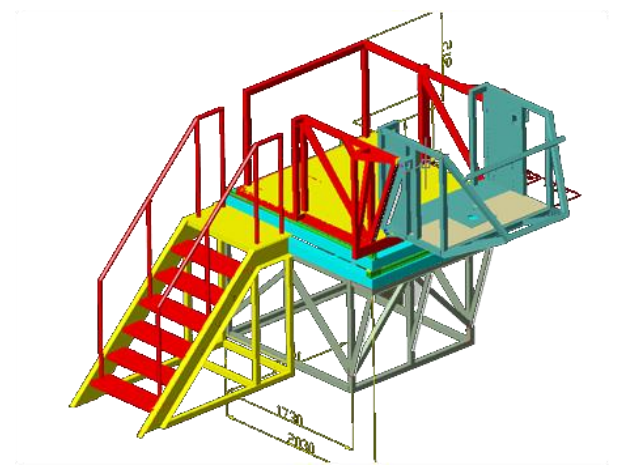

Figure 1. Mechanical Structure of Motion Platform 


\section{Wash-out algorithm of motion platform control}

\subsection{Algorithm Framework}

The motion platform mentioned in this work is mainly used for simulating the loading and unloading process of the container crane, which will generate the motion perception during the movement of crane and trolley. The driver control the simulator with the state of crane and training task, and the input signal into the wash-out algorithm is calculated from the kinetic model of crane in real time, such as linear acceleration and linear velocity. Generally speaking, human receive the motion by our vestibular system, and so the cab's center of mass should be translated into the driver's vestibular system. The wash-out algorithm of the platform movement control transforms the motion of the crane into the motion cues we can percept. The elongation of electric cylinder is determined by the inverse solution of the motion cues, to drive the movement of the motion platform. The wash-out algorithm of motion platform movement control is shown as Figure 2.

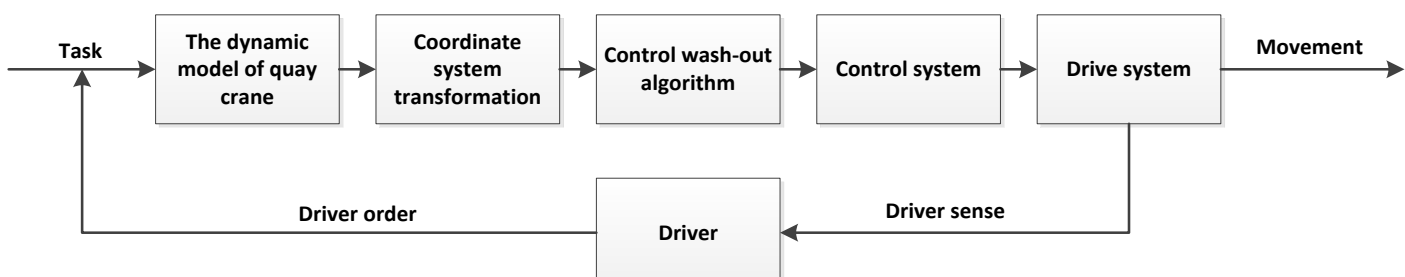

Figure 2. Wash-Out Algorithm Framework of Motion Platform Control

\subsection{Wash-out Algorithm Principle}

Human's sense of inertia motion is judged by the special force, which is perceived by vestibule. Special force is the vector difference between the inertial acceleration and gravitational acceleration $(\vec{f}=\vec{a}-\vec{g})$. The special force $f_{A A}$ is scaled changing by a limited scale module, and so the filter signal amplitude can be guaranteed that less than the selected amplitude. In the translation motion, the output $\mathrm{f}_{1}$ can be obtained:

In generally, $\mathrm{K}$ is selected as:

$$
\mathrm{f}_{1}=\mathrm{K}\left(\mathrm{f}_{\mathrm{AA}}+\mathrm{g}\right)-\mathrm{g}(1-1)
$$

$$
\mathrm{K}=\left[\begin{array}{ccc}
\mathrm{k}_{\mathrm{x}} & 0 & 0 \\
0 & \mathrm{k}_{\mathrm{y}} & 0 \\
0 & 0 & \mathrm{k}_{\mathrm{z}}
\end{array}\right]
$$

$\mathrm{k}_{\mathrm{x}}, \mathrm{k}_{\mathrm{y}}, \mathrm{k}_{\mathrm{z}}$ are scale coefficients in the matrix, and their value is confirmed by $f_{\text {AA. }}$.

If the system acceleration is filtered in the motion platform coordinate system, the motion platform can't move slowly return to the initial position. The platform can't complete next task after a movement is finished. In order to finish the filter process, the motion platform coordinate has to be translated into global coordinate system. Special force $\mathrm{f}_{2}$ can be obtained from $\mathrm{f}_{1}$ by using transformation matrix $\mathrm{L}_{\mathrm{s}}$, then plus the vector of gravity, the needed motion acceleration $\mathrm{a}_{2}$ with respect to Special force $\mathrm{f}_{2}$ can be obtained. The low-frequency signal, which would lead to the motion platform out of the guideway, is removed, and then the translation acceleration of motion platform $a_{h}$ can be obtained. The movement signal can be obtained with twice integral of acceleration signal and drive the platform moving. 
The high frequency part is filtered out by the low-pass filter, and then the angle $\beta_{\mathrm{L}}^{\prime}$, which the platform is needed for the movement $\mathrm{f}_{\mathrm{L}}$, can be obtained by using the angle coordinate. In order to guarantee the angle $\beta_{\mathrm{L}}^{\prime}$ not beyond the feeling threshold value, the angle of inclination $\beta_{\mathrm{L}}$, which can be worked out by adding the limited angle, is needed for the motion platform actuation.

\subsection{Parameters}

The filter is the important part of the motion platform design. This paper is mainly focus on the high-pass filter and low-pass filter. The high-pass filter can remove the low frequency signal, which may lead to the motion platform out of the work space, destroying the motion platform. The high-pass filter includes high-pass translation channel and the high-frequency rotation channel. There is no rotational motion of container crane when working, so we pay more attention on the translation movement. Considered the motion characteristics of the container crane, we use twice-order or third-order polynomials for the filters design. The parameters of natural frequency and damping ratio can be obtained by simulation for the highpass filter, as shown in Table 3.

Table 3. Parameters of the High-Pass Filter

\begin{tabular}{c|ccc}
\hline filter & $\boldsymbol{\omega}_{\mathbf{b}}$ & $\boldsymbol{\omega}_{\mathbf{n}}$ & $\boldsymbol{\varsigma}$ \\
\hline High-pass & 0.2 & 2.5 & 1 \\
\hline
\end{tabular}

As the rail can't provide an enough movement space, the simulator can't simulate a sustained acceleration. In order to realize the sustained movement sense, the simulator algorithm need to accomplish this process by using gravity vector's components of lateral axis and longitudinal axis under simulator coordinate system. The high-frequency signal of special force is removed by low-pass channel. The low-frequency signal, which would cause violent action and can't be implemented, is reserved.

Table 4 presents the parameters of the low filter.

Table 4. Parameters of the Low-Pass Filter

\begin{tabular}{c|cc}
\hline filter & $\boldsymbol{\omega}_{\mathbf{n}}$ & S \\
\hline Low-pass & 5.0 & 1 \\
\hline
\end{tabular}

To prevent feeling the rotation obviously, it is necessary to limit the angular velocity less than the human threshold, which close to $3^{\circ} / \mathrm{s}$.

\section{Performance Analysis}

We chose the trolley's movement as an example to analyze the performance. The following parameters were assumed for high-pass filter. As the working-condition of trolley movement, the trolley did uniform acceleration motion for $10 \mathrm{~s}$, and then did uniform motion. The acceleration was $2.4 \mathrm{~m} / \mathrm{s}$. The damping ratio of the simulation filter was 1 . The initial frequency was 2.5 . 


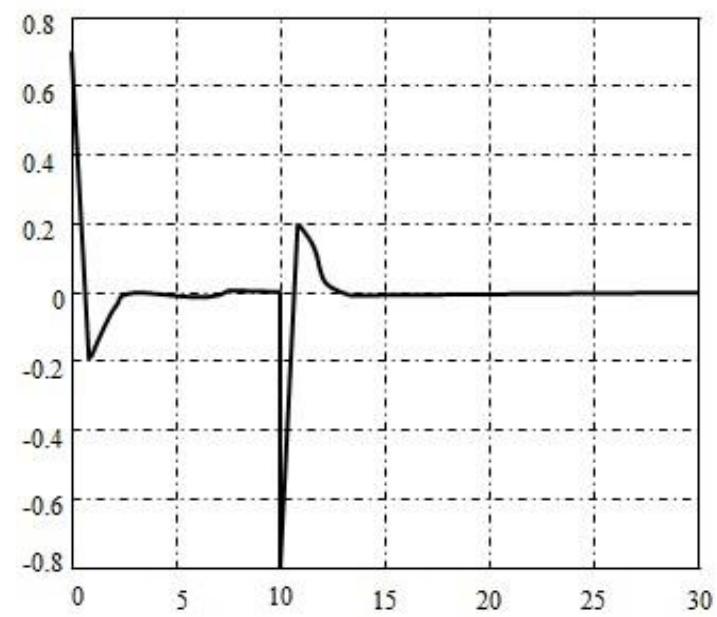

Figure 3. The Acceleration Curve of Trolley Movement

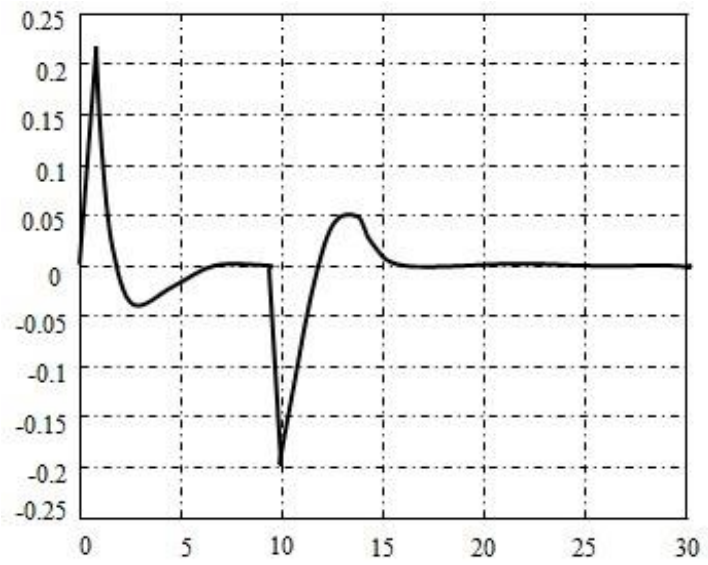

Figure 4. The Velocity Curve of Trolley Movement

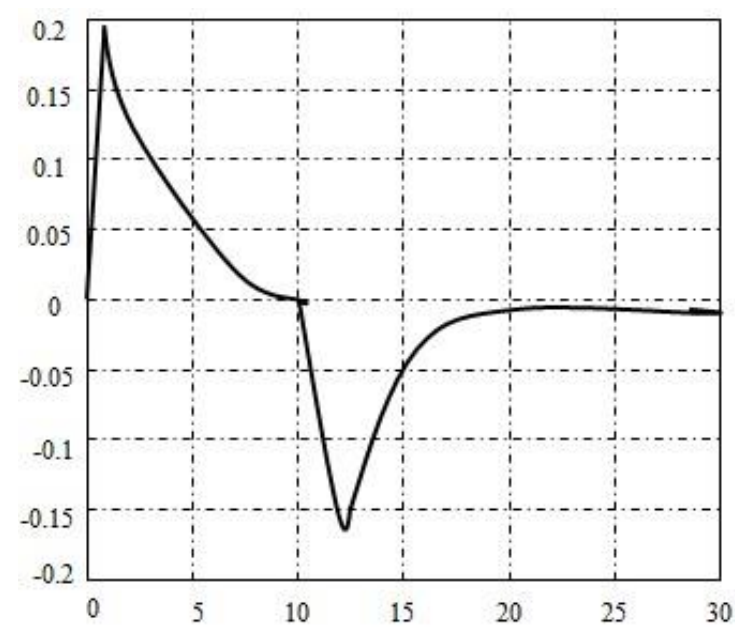

Figure 5. The Displacement Curve of Trolley Movement 


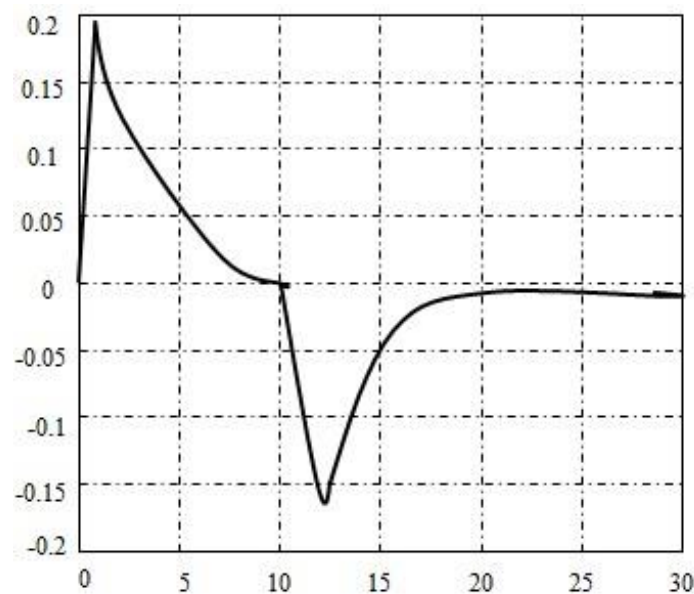

Figure 6. The Velocity Curve of Platform Tilt Channel

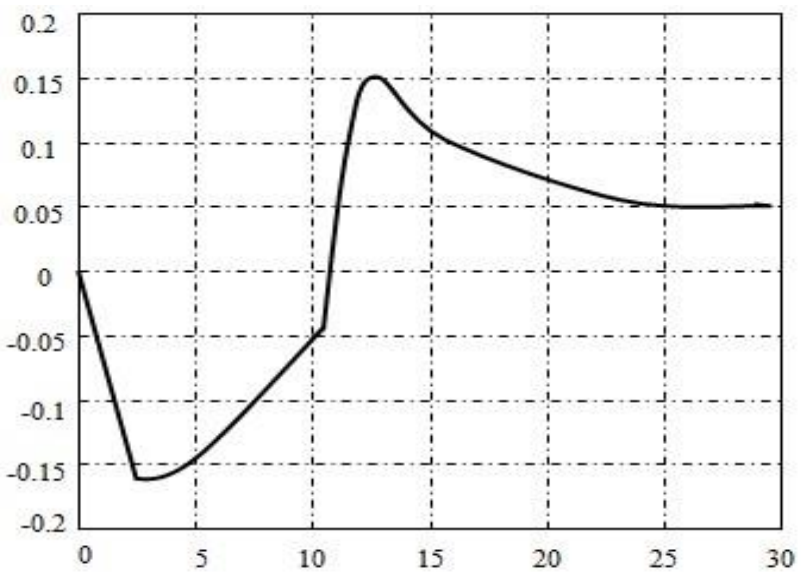

Figure 7. The Displacement Curve of Platform Tilt Channel

Figure 7 shows that the translation movement of motion platform just simulates the high-frequency of input signal. The low-frequency signal was applied to implement the pitch of motion platform by tilt compensation. Figure 5 shows that the motion platform didn't return to the initial position. As the order of the highpass filter is low, the motion platform would slowly return to the initial position when the order of the high-pass filter is greater than or equal 3. Figure 7 shows that the driver in simulator is the same movement sense as the driver in the real quay crane. The results demonstrate that the real movement sense can be simulated by wash-out algorithm. There are differences between them, because of the different algorithms and parameters.

\section{Conclusion}

Motion platform is the vital method to increase the fidelity and simulate effects of container crane simulator. It is also the necessary part of high-end simulator. Therefore this paper studied the motion platform design methods of container crane simulator according to the constitution of mechanism and performance parameter of container crane simulator. This project also studied the structural analysis, the forms of motion and the movement rule of the motion platform. The form of the structure and the range of motion can be determined according to the structural analysis and design of the selected quay crane movement rule. The control wash-out algorithm framework of suitable crane operating simulator was improved. The parameters of 
the filter and the validity of algorithm were determined according to the simulation. This motion platform has high control accuracy, stable working ability and thorough monitoring function, which can simulate different operating condition of container crane and satisfy the operating requirement.

\section{Acknowledgments}

This work sponsored by Shanghai Top Academic Discipline Project-management science \& engineering, National Natural Science Foundation project(71101090), Ministry of Transport Research Projects (2012-329-810-180), Shanghai Municipal Education Commission Project(12ZZ148, 13YZ080).

\section{References}

[1] X.L. Wang, "Study on movement perception simulation system for high speed train driving simulator", Southwest Jiaotong University, Chengdu, (2009).

[2] R. Graf and R. Dillmann, "Active acceleration compensation using a Stewart-platform on a mobile robot", Proceedings of the second EUROMICRO workshop on Advanced Mobile Robots, Brescia, Italy, (1997), pp.59-64.

[3] R.J. Telban, "New human-centered linear and nonlinear motion cueing algorithms for control of simulator motion systems", Binghamton University, Binghamton, (2003).

[4] C.-S. Liao, C.-F. Huanc and W.-H.Chieng, "A Novel Washout Filter Design for a Six Degree-ofFreedom Motion Simulator", JSME International Journal, vol.27, no.2, (2004), pp.626-636.

[5] A. Naseri and P. Grant, "An improved adaptive motion drive algorithm", AIAA Modeling and Simulation Technologies conference and Exhibit, San Francisco, USA, (2005), pp.1-9.

[6] X.L. Wang, L.L. Li and W.H. Zhang, "Effect of Washout Locations on the Fidelity of Locomotive Driving Simulator", Machinery \& Electronics, (2007), vol.4, pp.21-24.

[7] Y.I. Tang, X.L. Zhang and J.Z. Wang, "Realization of Digital Filter Based on Poprioceptive Simulation Algorithm for Driving Simulator", Computer Simulation, vol.26, no.7, (2009), pp.270-273.

[8] C.Y. Wei, X.J. Zhou and Y.D. Wei, "Washout motion simulation in 6-DOF parallel platform based on Vortex", Journals of Zhejiang University, vol.46, no.8, (2012), pp.1390-1396.

[9] J. Gao, "Research on fidelity of motion cueing system in flight simulator", Haerbing Industry University, Haerbing, (2013).

[10] Y. Lei and Y.Y. Li, "Development and control of broken-strap monitoring system for newspaper flexiroll machines", International Journal of Automation and Control, vol.6, no.23, (2012), pp.81-103.

\section{Authors}

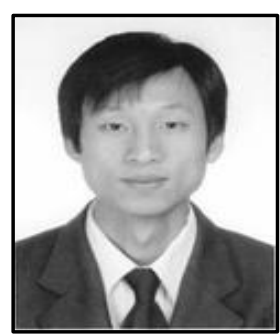

Houjun Lu, he is now pursuing studies in machinery design and theory major, and my research interests include virtual reality technology, driving simulation, closed-loop system evaluation. 